Trinity University

Digital Commons @ Trinity

Physics and Astronomy Faculty Research

Physics and Astronomy Department

$10-2018$

\title{
X-ray Swift Observations of SN 2018cow
}

\author{
LE. Rivera Sandoval
}

T J. Maccarone

A Corsi

P J. Brown

David Pooley

Trinity University, dpooley@trinity.edu

See next page for additional authors

Follow this and additional works at: https://digitalcommons.trinity.edu/physics_faculty

Part of the Physics Commons

\section{Repository Citation}

Rivera Sandoval, L.E., Maccarone, T.J., Corsi, A., Brown, P.J., Pooley, D., \& Wheeler, J.C. (2018). X-ray Swift observations of SN 2018cow. Monthly Notices of the Royal Astronomical Society: Letters, 480(1), L146-L150. doi: 10.1093/mnrasl/sly145

This Pre-Print is brought to you for free and open access by the Physics and Astronomy Department at Digital Commons @ Trinity. It has been accepted for inclusion in Physics and Astronomy Faculty Research by an authorized administrator of Digital Commons @ Trinity. For more information, please contact jcostanz@trinity.edu. 


\section{Authors}

L E. Rivera Sandoval, T J. Maccarone, A Corsi, P J. Brown, David Pooley, and J C. Wheeler 


\title{
X-ray Swift observations of SN 2018cow
}

\author{
L.E. Rivera Sandoval ${ }^{1 \star}$, T.J. Maccarone ${ }^{1}$, A. Corsi ${ }^{1}$, P.J. Brown ${ }^{2}$, D. Pooley ${ }^{3}$,
}

\author{
J.C. Wheeler ${ }^{4}$ \\ ${ }^{1}$ Department of Physics and Astronomy, Box 41051, Science Building, Texas Tech University, Lubbock, TX 79409-1051, USA \\ ${ }^{2}$ George P. and Cynthia Woods Mitchell Institute for Fundamental Physics 83 Astronomy, Texas A. 83 M. \\ University, Department of Physics and Astronomy, 4242 TAMU, College Station, TX 77843, USA \\ ${ }^{3}$ Department of Physics and Astronomy, Trinity University, San Antonio, TX, USA ; Eureka Scientific, Inc., USA \\ ${ }^{4}$ Department of Astronomy, University of Texas at Austin, Austin, TX, USA
}

18 July 2018

\begin{abstract}
Supernova (SN) 2018cow is an optical transient detected in the galaxy CGCG 137-068. It has been classified as a SN due to various characteristics in its optical spectra. The transient is also a bright X-ray source. We present results of the analysis of $\sim 62 \mathrm{ks}$ of X-ray observations taken with the Neil Gehrels Swift Observatory over 27 days. We found a variable behavior in the $0.3-10 \mathrm{keV}$ X-ray light curve of SN 2018cow, with variability timescales of days. The observed X-ray variability could be due to the interaction between the SN ejecta and a non-uniform circum-stellar medium, perhaps related to previous mass ejections from a luminous-blue-variable-like progenitor.
\end{abstract}

Key words: supernovae: individual: SN 2018cow - X-rays: stars - gamma-ray burst: general

\section{INTRODUCTION}

SN 2018cow (also known as ATLAS18qqn) is an optical transient detected on 16 June 2018 (10:35:02 UT) with the Asteroid Terrestrial-impact Last Alert System (ATLAS) located at Haleakala and Mauna Loa, Hawaii, USA (Smartt et al. 2018).

The position of the transient $\left(\mathrm{RA}=16^{\mathrm{h}} 16^{\mathrm{m}} 00^{\mathrm{s}} .22\right.$, Dec $=+22^{\circ} 16^{\prime} 04^{\prime \prime} .8$; J2000) is coincident with that of the galaxy CGCG 137-068, which is located at a redshift of $0.014^{1}$, corresponding to a luminosity distance of $59.7 \pm$ $4.2 \mathrm{Mpc}$ (assuming $H_{0}=73.0 \pm 5 \mathrm{~km} \mathrm{sec}^{-1} \mathrm{Mpc}^{-1}$ ). SN 2018cow was initially thought to be a cataclysmic variable (CV) star (Smartt et al. 2018). However, spectral observations taken on 18 June 2018 UT found a featureless spectrum (Perley 2018). Observations taken on 19 June 2018 UT revealed the presence of Ca II H\&K absorption features at the redshift of CGCG 137-068, thus confirming that SN 2018cow was in fact an extragalactic transient (Jones et al. 2018). The first X-ray observations of SN 2018cow taken with the Neil Gehrels Swift Observatory also on 19 June 2018 (starting at 10:34:53 UT; Rivera Sandoval \& Maccarone 2018a,b) showed that the object had a $0.3-10 \mathrm{keV}$

\footnotetext{
* E-mail: liliana.rivera@ttu.edu

1 Value obtained from the NASA/IPAC extragalactic database http://ned.ipac.caltech.edu/forms/byname.html.
}

flux of $(2.6 \pm 0.3) \times 10^{-11} \mathrm{erg} \mathrm{cm}^{-2} \mathrm{~s}^{-1}$, and a hard spectrum with a photon index of $\Gamma=1.6 \pm 0.1$. These observations also helped to discard the hypothesis that SN 2018cow was a $\mathrm{CV}$, since at a distance limit of $\sim 700 \mathrm{pc}$ derived from its optical luminosity ${ }^{2}$ (V=13.8 mags, Rivera Sandoval \& Maccarone 2018a), the object would have an X-ray luminosity $L_{X} \sim 1.5 \times 10^{33} \mathrm{erg} \mathrm{s}^{-1}$, which is much higher than those of typical CVs in outburst $\left(L_{X} \lesssim 10^{32} \mathrm{erg} \mathrm{s}^{-1}\right.$, see e.g. Ramsay et al. 2001; Saitou et al. 2012). Instead, these X-ray observations suggested that the object could be a Gamma-Ray Burst (GRB) afterglow. GRB afterglows have $\mathrm{X}$-ray luminosities in the range $10^{43}-10^{46} \mathrm{erg} \mathrm{s}^{-1}$ at $\sim 24 \mathrm{hrs}$ after trigger (D'Avanzo et al. 2012), and SN 2018cow at the distance of $\sim 60 \mathrm{Mpc}$ would have an X-ray luminosity $L_{X} \sim 1.15 \times 10^{43} \mathrm{erg} \mathrm{s}^{-1}$. X-ray luminosities of Type $\mathrm{Ib} /$ Ic supernovae after few days of the explosion are below $10^{42} \mathrm{erg} \mathrm{s}^{-1}$ (Drout et al. 2016), slightly lower than the observed luminosity of the transient. Alternatively, the X-rays could merely represent a rather extreme shock breakout.

No $\gamma$-rays in the $14-195 \mathrm{keV}$ range were detected with Swift/BAT around the time of optical discovery (Lien et al. 2018). Fermi/GBM did not detect activity spatially coincident with SN 2018cow (Dal Canton et al. 2018) in the period

\footnotetext{
2 Assuming a typical absolute optical magnitude for CVs in outburst $M_{V} \sim 4.5$ (Warner 1987).
} 
13-16 June 2018, but it is possible that the event occurred outside the field of view of such telescope because the sky region of the transient was observed only $54 \%$ of the time during that period (this could be the case for Swift/BAT as well). Additionally, no detection at $\mathrm{TeV}$ energies were made in the period 13-16 June 2018 UT with $H A W C$ in the direction of the transient (Garcia-Gonzalez et al. 2018). Ultraviolet observations carried out on 19 June 2018 (10:40:40 UT) with the UVOT instrument on board of Swift, showed that SN 2018cow was also bright at such wavelengths, with Vega magnitude of $11.70 \pm 0.01$ (Rivera Sandoval \& Maccarone 2018a).

SN 2018cow was also identified at millimetre wavelengths with NOEMA on 20 June 2018 UT, with a flux of $\sim 6 \mathrm{mJy}$ at $90 \mathrm{GHz}$ (de Ugarte Postigo et al. 2018). On 22 June 2018 UT the object had a flux density of $\sim 0.5 \mathrm{mJy}$ determined using data from AMI-LA at a central frequency of $15.5 \mathrm{GHz}$ (Bright et al. 2018). ATCA detected the transient at $34 \mathrm{GHz}$ with a flux of $\sim 5.6 \mathrm{mJy}$ on 26 June 2018 UT (Dobie et al. 2018a). The flux in that band increased to $\sim 7.6 \mathrm{mJy}$ two days later (Dobie et al. 2018b).

From spectroscopic observations of SN 2018cow carried out on 20 June 2018 UT with the Xinglong-2.16m, Xu et al. (2018) reported a broad bump or absorption feature (He I [3889] if absorption) and a broad feature at $5040 \AA$ that could be the signature of high velocity blending as in broadline Type Ic supernovae (SNe BL-Ic). Izzo et al. (2018) reported further spectroscopic follow up with ISIRIS/GTC on 21 June finding broad undulations similar to SNe BL-Ic, though without a direct match, though absorption blueward of a $5460 \AA$ peak could be FeII at $20,000 \mathrm{~km} \mathrm{~s}^{-1}$. Srivastav et al. (2018) reported on the evolution of the 4500/5500 $\AA$ feature, finding it to have strengthened until 5 days after discovery and then weaking to almost disappear in Liverpool Telescope spectra taken on 24 June. This would be unusual for a SN emerging from a fading afterglow and/or shock breakout. From spectra taken on 8 July UT, 22 days after discovery, Benetti et al. (2018) identified emission features that could be associated to He lines, thus re-classifying SN 2018cow as a Type Ib SN.

In this letter we present results of the analysis of 27 days of continuous X-ray follow-up observations of SN 2018cow with Swift in the $0.3-10 \mathrm{keV}$ energy band, starting approximately three days after its discovery.

\section{OBSERVATIONS}

The $\sim 62$ ks of X-ray Swift observations analyzed in this letter cover the period from 19 June 2018 to 13 July 2018 UT. These are a total of 72 observations with exposure times ranging from $\sim 200 \mathrm{~s}$ to $\sim 3000 \mathrm{~s}$. The cadence of the X-ray observations is variable, but data were taken at least once per day. We discarded the observation taken on 21 June 2018 at 11:47:14 UT, as the data were heavily affected by a hot column right at the SN 2018 cow position.

All observations were reduced using the Swift software $^{3}$. First, we reprocessed the data using the routine XRTPIPELINe. Then, the routines Xselect and Ximage were

3 http://www.swift.ac.uk/analysis/xrt/setup.php

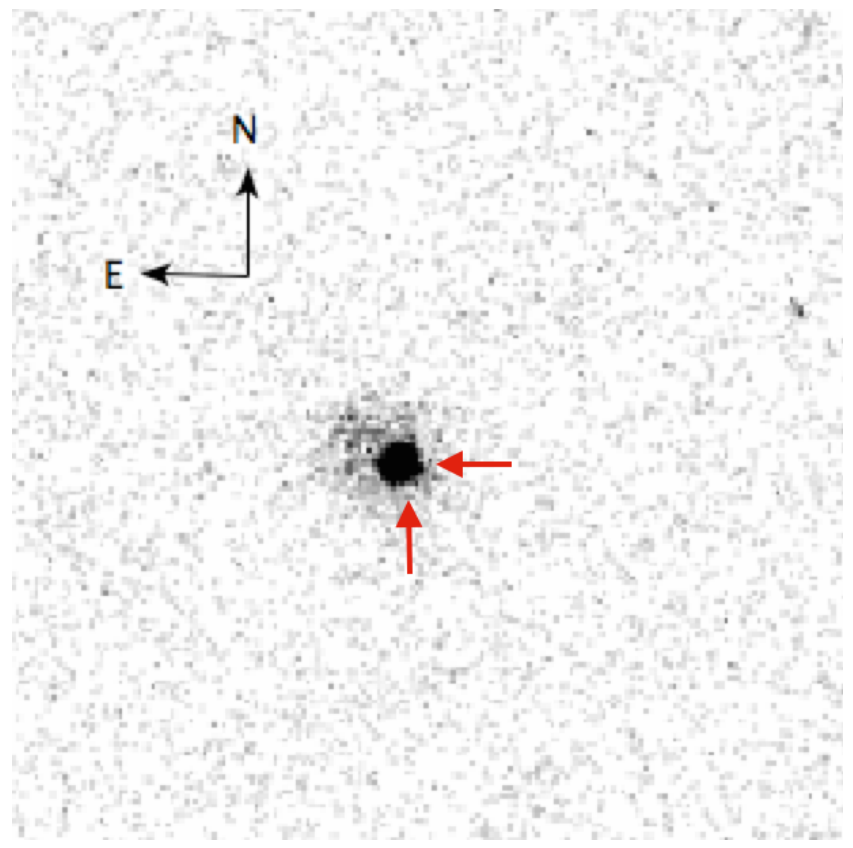

Figure 1. Swift/UVOT ultraviolet image of SN 2018cow taken during the X-ray luminosity increase on 29 June 2018 in the filter UVM2. The extended emission surrounding the object is likely due to its host galaxy. The image is $3^{\prime} \times 3^{\prime}$. No increase in the UVM2 magnitude was observed during this episode. The arrows indicate the position of SN 2018cow.

used to measure count rates in the $0.3-10 \mathrm{keV}$ energy band. We adopted a threshold signal-to-noise ratio of 3 for detections. To determine rate correction factors due to bad columns we used the routine XRTLCCORR.

For the spectral analysis we followed the SwIFT thread ${ }^{4}$. We used XRTMKARF to create the ancillary response files. For the spectral fitting we used the software package XSPEC (v12.9.1 Arnaud 1996). To model the hydrogen column density $\mathrm{N}_{H}$ we used the model tbnew_gas ${ }^{5}$ with WILM abundances (Wilms et al. 2000) and VERN cross-sections (Verner et al. 1996). We fitted our spectra with a power-law model (PEGPWRLW). For the spectrum presented in Figure 3 the data were grouped to have at least 15 counts per bin.

\section{RESULTS}

In Figure 2 we show the Swift 0.3-10 keV light curve of SN 2018cow from 19 June 2018 UT to 13 July 2018 UT. Data behind this Figure are reported in Table A1. We see that SN 2018cow shows a variable behavior on time scales of days. There are multiple re-brightenings after the first detection on 19 June 2018 UT. The most significant increase in the Xray count rate is the one detected on 29 June 2018 (09:21:01 $\mathrm{UT}^{6}$ ), with a rate of $0.35 \pm 0.05$ counts s$^{-1}$ (see also Figure 1 ). Fitting an absorbed power-law model to that observation,

\footnotetext{
4 http://www.swift.ac.uk/analysis/xrt/spectra.php

5 http://pulsar.sternwarte.uni-erlangen.de/wilms/research/tbabs/

6 This observation was not affected by a hot column, unlike the longer observation recorded at 09:24:14 UT.
} 


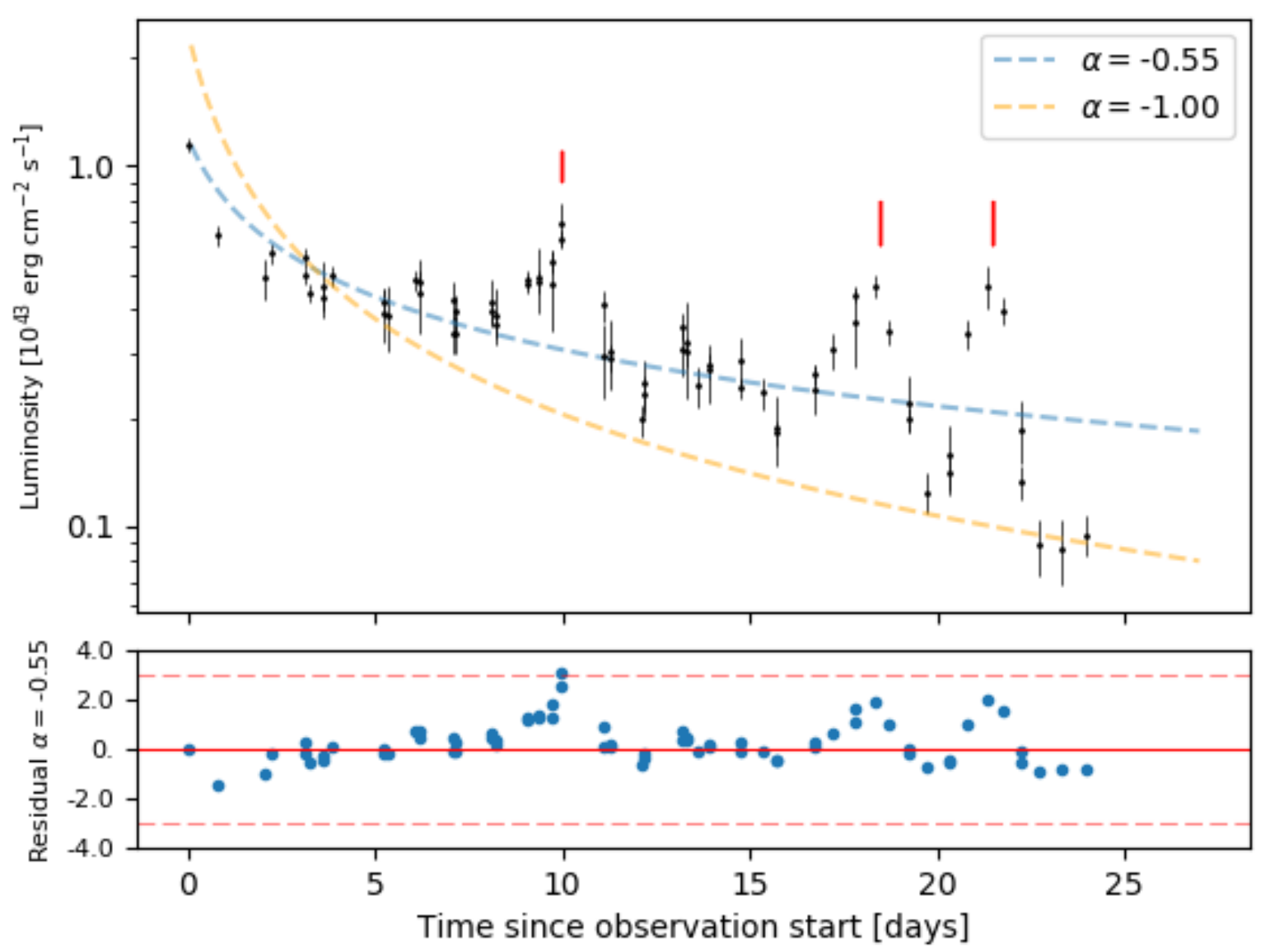

Figure 2. X-ray Swift light curve of SN 2018cow from 19 June 2018 (10:34:53 UT) to 13 July 2018 (09:56:36 UT) in the 0.3-10 keV energy band. A power-law fit with $\alpha=-0.55 \pm 0.01$ (where $L_{X} \propto t^{\alpha}$ ) is shown (blue line). Significant deviations appear during the episodes in which the X-ray luminosity increased. For comparison, we also plotted a fit with $\alpha=-1$ (orange line). Residuals normalized to the rms error are shown in the bottom part of the plot. The dashed lines indicate the $3 \sigma$ level. The presence of consecutive excesses, increases the detection significance of the events (indicated by the red vertical lines). A table with the data is given in the Appendix.

Rivera Sandoval \& Maccarone (2018c) determined an X-ray flux in the $0.3-10 \mathrm{keV}$ of $(1.8 \pm 0.7) \times 10^{-11} \mathrm{erg} \mathrm{cm}^{-2} \mathrm{~s}^{-1}$ and $\Gamma=$ $1.2 \pm 0.8$ which represented a flux increase of approximately a factor of two with respect to the observations taken on 21 June 2018 (16:37:11 UT), when the object had a flux of $(1.2 \pm 0.2) \times 10^{-11} \mathrm{erg} \mathrm{cm}^{-2} \mathrm{~s}^{-1}$ and $\Gamma=1.8 \pm 0.3$ (Sandoval et al. 2018).

The X-ray spectrum of SN 2018cow in the $0.3-10 \mathrm{keV}$ band can be described by a power-law model with $\Gamma \lesssim 2$ (Figure 3) at all epochs. There is no evidence for spectral evolution in the $0.3-10 \mathrm{keV}$ band since discovery, and no evidence for significant spectral evolution during the X-ray increases (measurements are consistent within $90 \%$ errors). The value of the spectral index is in agreement with observations carried out with Chandra and NICER (Maccarone et al. 2018; Miller et al. 2018) and also with NuSTAR and INTEGRAL at higher energies (Margutti et al. 2018; Grefenstette et al. 2018; Savchenko et al. 2018). Though, indications of a hard component at energies above $15 \mathrm{keV}$ was found on 23 June 2018 (Margutti et al. 2018), which was not longer observed on 2 July 2018 (Grefenstette et al. 2018). The combined spectrum of SN 2018cow up to 13 July 2018 can be described with a $\Gamma \sim 1.6$.

Assuming a decay of the luminosity $L_{X} \propto t^{\alpha}$, we found that the temporal evolution of the $0.3-10 \mathrm{keV}$ luminosity can be best fitted with a power-law index $\alpha=-0.55 \pm 0.01$ (figure 2), which is not consistent with the one found by INTEGRAL, where $\alpha=-1$ (Savchenko et al. 2018). The Xray increases shown in the light curve of SN 2018cow seem to significantly deviate from that fit. The presence of consecutive excesses in the residuals, give us confidence on the significance of these events.

\section{DISCUSSION}

SN 2018cow has been classified as a candidate SN due to its optical spectral properties(Xu et al. 2018; Perley et al. 


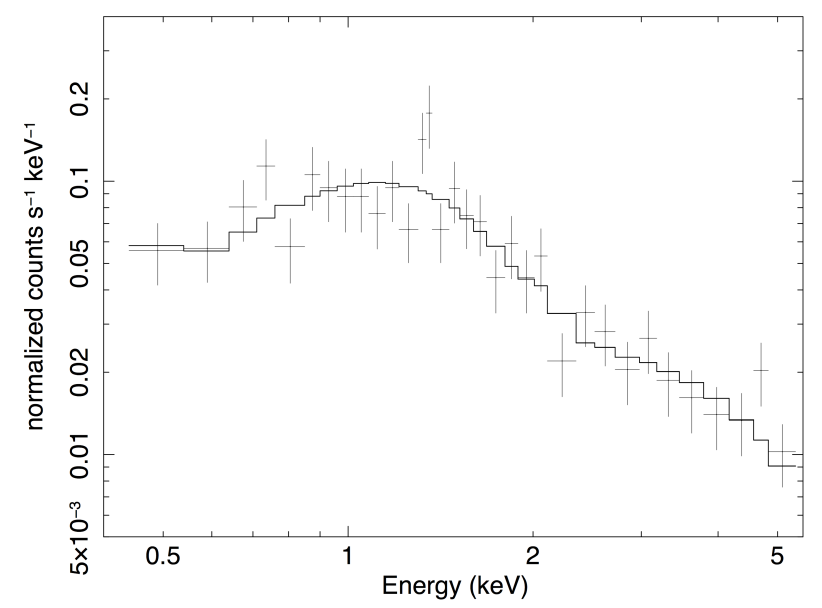

Figure 3. X-ray spectrum of SN 2018 cow in the $0.3-10 \mathrm{keV}$ energy band, using a $\sim 3 \mathrm{ks}$ Swift observation taken on 7 July 2018. The spectrum can be described with a photon index $\Gamma=1.4 \pm 0.2$. The X-ray flux was $(1.1 \pm 0.1) \times 10^{-11} \mathrm{erg} \mathrm{cm}^{-2} \mathrm{~s}^{-1}$. Quoted errors are at $90 \%$ confidence level.

2018; Benetti et al. 2018). However, an unambiguous classification remains to be established. If indeed SN 2018cow is a $\mathrm{SN}$, its variable X-ray behavior (Fig. 2) could be explained as due to the interaction of the SN shock with non-uniform circum-stellar material (CSM), perhaps related to eruptive, Luminous Blue Variable (LBV)-like mass-loss from the presupernova progenitor. Assuming a wind velocity $\mathrm{v}_{\text {wind }} \sim$ $1000 \mathrm{~km} \mathrm{~s}^{-1}$ (typical for Type Ib/Ic SN; Wellons et al. 2012), and a $\mathrm{SN}$ expansion velocity $\mathrm{v}_{S N} \sim 20,000 \mathrm{~km} \mathrm{~s}^{-1}$ as determined for SN 2018cow from optical spectroscopy (Izzo et al. 2018), then the $\sim 10$ days between the first two X-ray peaks (linked to interactions between the SN and the LBV-like ejecta) would correspond to LBV-like eruptions spaced by $\sim 200$ days. This value is consistent with pulsations of some LVB stars (Lamers et al. 1998). Note, however, that the mass-ejections from the progenitor could have been neither at a constant rate nor at a constant velocity. This would help explain the variable frequency of the observed increases in the X-ray light curve, such as the occurrence of the last one.

Flux increases in the X-ray light curves of Type Ib/Ic $\mathrm{SNe}$ are not commonly observed within few weeks after the explosion. Usually these light curves follow smooth decays (e.g. Modjaz et al. 2009; Campana et al. 2006a). However, there are records of X-ray flux variations in some SNe due to strong interaction with the CSM (e.g. Immler et al. 2008), which supports the idea that the variations observed in the X-ray light curve of SN 2018cow could be indeed caused by a similar mechanism, though with different factors affecting the timescale of occurrence of the re-brightening episodes. GRB X-ray afterglows also show increases in their light curve. However, they occur at time scales much shorter than those observed for the SN 2018cow (see e.g. Campana et al. 2006b; Ruffini et al. 2018).

In order to determine the nature of SN 2018cow Xray emission, we estimated the radio-to-X-ray spectral index comparing the $34 \mathrm{GHz}$ flux $(\sim 5.6 \mathrm{mJy}$; Dobie et al. 2018a) measured on 26 June 2018 (09:00-14:00 UT), to the Swift X-ray flux of two measurements taken the same day within that time interval (13:06:00-13:21:53 UT, $F_{X}=$ $\left.9.2 \times 10^{-12} \mathrm{erg} \mathrm{cm}^{-2} \mathrm{~s}^{-1} / 2.4 \times 10^{17} \mathrm{~Hz} \approx 4 \times 10^{-6} \mathrm{mJy}\right)$. We obtained $\Gamma_{r} \approx 1.9$. This value is in agreement with the $\mathrm{X}-$ ray spectral index obtained from the Swift data. It is also in agreement with results for other CSM-interacting BL-Ic SNe (Corsi et al. 2014) in which the X-rays seem to have a synchrotron origin. This suggests that a similar mechanism may be powering SN 2018cow X-ray emission.

Follow-up observations of SN 2018cow at all the wavelengths will likely allow to obtain more insights about its nature. However, whatever its classification, SN 2018cow is already an outstanding object due to the time variability of its X-ray light curve, and to its peculiar multi-wavelength behavior in general.

\section{ACKNOWLEDGEMENTS}

We thank the Swift team for their fast response to our multiple target of opportunity requests, which provided us with the data used in this paper, and additionally thank Jamie Kennea and Kim Page for further useful advice regarding the Swift data. Thanks also to Yuri Cavecchi for comments on the manuscript.

\section{REFERENCES}

Arnaud K. A., 1996, in Jacoby G. H., Barnes J., eds, Astronomical Society of the Pacific Conference Series Vol. 101, Astronomical Data Analysis Software and Systems V. p. 17

Benetti S., Pastorello A., Cappellaro E., Turatto M., Tomasella L., Fiore A., Reguitti A., Gromadzki M., 2018, The Astronomer's Telegram, 11836

Bright J., Horesh A., Fender R., Motta S., Sfaradi I., Titterington D., Perrott Y., Ho A., 2018, The Astronomer's Telegram, 11774

Campana S., et al., 2006a, Nature, 442, 1008

Campana S., et al., 2006b, A\&A, 454, 113

Corsi A., et al., 2014, ApJ, 782, 42

D'Avanzo P., et al., 2012, MNRAS, 425, 506

Dal Canton T., et al., 2018, The Astronomer's Telegram, 11793

Dobie D., Ravi V., Ho A., Kasliwal M., Murphy T., 2018a, The Astronomer's Telegram, 11795

Dobie D., Ravi V., Ho A., Kasliwal M., Murphy T., 2018b, The Astronomer's Telegram, 11818

Drout M. R., et al., 2016, ApJ, 821, 57

Garcia-Gonzalez J. A., Gonzalez M., Martinez I., HAWC Collaboration 2018, The Astronomer's Telegram, 11792

Grefenstette B., Margutti R., Chornock R., Metzger B., Migliori G., 2018, The Astronomer's Telegram, 11813

Immler S., et al., 2008, ApJ, 674, L85

Izzo L., et al., 2018, The Astronomer's Telegram, 11753

Jones D. O., Rojas-Bravo C., Dimitriadis G., Pan Y.-C., Foley R. J., 2018, The Astronomer's Telegram, 11736

Lamers H. J. G. L. M., Bastiaanse M. V., Aerts C., Spoon H. W. W., 1998, A\&A, 335, 605

Lien A. Y., et al., 2018, The Astronomer's Telegram, 11782

Maccarone T. J., Sandoval L. R., Corsi A., Pooley D., Knigge C., 2018, The Astronomer's Telegram, 11779

Margutti R., Chornock R., Laskar T., Fong W., Grefenstette B., Risaliti G., 2018, The Astronomer's Telegram, 11775

Miller J. M., et al., 2018, The Astronomer's Telegram, 11773

Modjaz M., et al., 2009, ApJ, 702, 226

Perley D., 2018, The Astronomer's Telegram, 11732 
Perley D. A., Blagorodnova N., Neill J. D., Walters R., 2018, The Astronomer's Telegram, 11776

Ramsay G., et al., 2001, A\&A, 365, L294

Rivera Sandoval L. E., Maccarone T., 2018a, The Astronomer's Telegram, 11737

Rivera Sandoval L. E., Maccarone T., 2018b, The Astronomer's Telegram, 11739

Rivera Sandoval L. E., Maccarone T., 2018c, The Astronomer's Telegram, 11801

Ruffini R., et al., 2018, ApJ, 852, 53

Saitou K., Tsujimoto M., Ebisawa K., Ishida M., 2012, PASJ, 64, 88

Sandoval L. E. R., Maccarone T., Brown P., 2018, The Astronomer's Telegram, 11761

Savchenko V., Ferrigno C., Kuulkers E., Goetz D., Bozzo E. e. a., 2018, The Astronomer's Telegram, 11843

Smartt S. J., et al., 2018, The Astronomer's Telegram, 11727

Srivastav S., Singh A., Kumar B., Kumar H., Bhalerao V., Anupama G. C., Sahu D. K., Bandyopadhyay A., 2018, The Astronomer's Telegram, 11766

Verner D. A., Ferland G. J., Korista K. T., Yakovlev D. G., 1996, ApJ, 465, 487

Warner B., 1987, MNRAS, 227, 23

Wellons S., Soderberg A. M., Chevalier R. A., 2012, ApJ, 752, 17

Wilms J., Allen A., McCray R., 2000, ApJ, 542, 914

Xu D., et al., 2018, The Astronomer's Telegram, 11740

de Ugarte Postigo A., et al., 2018, The Astronomer's Telegram, 11749

\section{APPENDIX A: SWIFT X-RAY LIGHT CURVE \\ DATA}

We present the Swift data used to generate figure 2 in Section 3 .

This paper has been typeset from a $\mathrm{T}_{\mathrm{EX}} \mathrm{L} \mathrm{LT}_{\mathrm{E}} \mathrm{X}$ file prepared by the author.
Table A1. Swift X-ray light curve of SN 2018cow in the 0.3$10 \mathrm{keV}$. The time since the first observation (19 June 2018 at 10:34:53 UT) is indicated in column 1 . We have used a factor of $1.97 \times 10^{43} \mathrm{erg} \mathrm{cm}^{-2} \mathrm{~s}^{-1}$ to convert the count rates to luminosities.

\begin{tabular}{ccc}
$\begin{array}{c}\mathrm{t} \\
\text { days })\end{array}$ & $\begin{array}{c}\text { Luminosity } \\
\left(10^{43} \mathrm{erg} \mathrm{cm}^{-2} \mathrm{~s}^{-1}\right)\end{array}$ & $\begin{array}{c}\sigma_{L_{X}} \\
\left(10^{43} \mathrm{erg} \mathrm{cm}^{-2} \mathrm{~s}^{-1}\right)\end{array}$ \\
\hline \hline 0.000 & 1.147 & 0.051 \\
0.781 & 0.642 & 0.041 \\
2.047 & 0.488 & 0.061 \\
2.252 & 0.573 & 0.036 \\
3.116 & 0.557 & 0.038 \\
3.116 & 0.496 & 0.028 \\
3.251 & 0.444 & 0.028 \\
3.639 & 0.460 & 0.081 \\
3.641 & 0.431 & 0.034 \\
3.838 & 0.496 & 0.030 \\
5.233 & 0.391 & 0.065 \\
5.236 & 0.419 & 0.038 \\
5.366 & 0.383 & 0.077 \\
6.099 & 0.484 & 0.032 \\
6.165 & 0.446 & 0.103 \\
6.168 & 0.474 & 0.036 \\
7.105 & 0.425 & 0.053 \\
7.110 & 0.344 & 0.041 \\
7.167 & 0.393 & 0.043 \\
7.170 & 0.342 & 0.041 \\
8.096 & 0.421 & 0.065 \\
8.099 & 0.393 & 0.030 \\
8.231 & 0.387 & 0.067 \\
8.234 & 0.363 & 0.030 \\
9.091 & 0.472 & 0.028 \\
9.093 & 0.486 & 0.030 \\
9.356 & 0.492 & 0.101 \\
9.358 & 0.480 & 0.036 \\
9.752 & 0.468 & 0.118 \\
9.754 & 0.543 & 0.038 \\
9.949 & 0.697 & 0.095 \\
9.951 & 0.628 &
\end{tabular}


Table A1. Continued

\begin{tabular}{ccc}
$\begin{array}{c}\mathrm{t} \\
(\text { days })\end{array}$ & $\begin{array}{c}\text { Luminosity } \\
\left(10^{43} \mathrm{erg} \mathrm{cm}^{-2} \mathrm{~s}^{-1}\right)\end{array}$ & $\begin{array}{c}\sigma_{L_{X}} \\
\left(10^{43} \mathrm{erg} \mathrm{cm}^{-2} \mathrm{~s}^{-1}\right)\end{array}$ \\
\hline \hline 11.078 & 0.296 & 0.069 \\
11.080 & 0.411 & 0.041 \\
11.277 & 0.306 & 0.067 \\
11.279 & 0.294 & 0.026 \\
12.143 & 0.197 & 0.020 \\
12.207 & 0.249 & 0.041 \\
12.209 & 0.231 & 0.030 \\
13.205 & 0.310 & 0.049 \\
13.209 & 0.359 & 0.032 \\
13.346 & 0.324 & 0.097 \\
13.348 & 0.304 & 0.030 \\
13.601 & 0.245 & 0.032 \\
13.941 & 0.271 & 0.049 \\
13.945 & 0.278 & 0.022 \\
14.731 & 0.290 & 0.043 \\
14.734 & 0.243 & 0.016 \\
15.339 & 0.235 & 0.024 \\
15.728 & 0.188 & 0.041 \\
15.730 & 0.182 & 0.014 \\
16.724 & 0.241 & 0.038 \\
16.727 & 0.265 & 0.018 \\
17.194 & 0.308 & 0.036 \\
17.788 & 0.369 & 0.093 \\
17.789 & 0.438 & 0.020 \\
18.329 & 0.464 & 0.034 \\
18.719 & 0.348 & 0.028 \\
19.253 & 0.221 & 0.039 \\
19.255 & 0.197 & 0.016 \\
19.714 & 0.124 & 0.016 \\
20.320 & 0.158 & 0.032 \\
20.322 & 0.140 & 0.018 \\
20.783 & 0.342 & 0.063 \\
21.333 & 0.462 & 0.032 \\
21.773 & 0.397 & 0.038 \\
22.242 & 0.186 & 014 \\
22.244 & 0.132 & \\
22.715 & 0.089 & 0.016 \\
23.315 & 0.087 & \\
23.973 & 0.095 & \\
& & \\
\hline
\end{tabular}

